\title{
Generalized entropy in expanded semigroups and in algebras with neutral element
}

\author{
Erkko Lehtonen • Agata Pilitowska
}

Received: 17 November 2011 / Accepted: 14 December 2013 / Published online: 22 January 2014

(C) The Author(s) 2014. This article is published with open access at Springerlink.com

\begin{abstract}
The complex product of (non-empty) subalgebras of a given algebra from a variety $\mathcal{V}$ is again a subalgebra if and only if the variety $\mathcal{V}$ has the so-called generalized entropic property. This paper is devoted to algebras with a neutral element or with a semigroup operation. We investigate relationships between the generalized entropic property and the commutativity of the fundamental operations of the algebra. In particular, we characterize the algebras with a neutral element that have the generalized entropic property. Furthermore, we show that, similarly as for $n$-monoids and $n$-groups, for inverse semigroups, the generalized entropic property is equivalent to commutativity.
\end{abstract}

Keywords Entropy · Generalized entropy · Neutral element · Associativity · Symmetry $\cdot$ Inverse semigroup $\cdot n$-Semigroup

Communicated by Mikhail Volkov.

While working on this paper, the second author was supported by the Statutory Grant of Warsaw University of Technology 504G11200054000.

E. Lehtonen

Computer Science and Communications Research Unit, University of Luxembourg, 6, rue Richard Coudenhove-Kalergi, 1359 Luxembourg, Luxembourg

e-mail: erkko@campus.ul.pt

Present address:

E. Lehtonen

Centro de Álgebra da Universidade de Lisboa, Avenida Professor Gama Pinto 2, 1649-003 Lisbon, Portugal

A. Pilitowska (凶)

Faculty of Mathematics and Information Science, Warsaw University of Technology, Koszykowa 75, 00-662 Warsaw, Poland

e-mail: apili@mini.pw.edu.pl 


\section{Introduction}

Let $A$ be a nonempty set. Throughout this paper, we consider operations on $A$, i.e., maps $f: A^{n} \rightarrow A$, where $n$ is a nonnegative integer, called the arity of $f$. We say that two operations $f: A^{n} \rightarrow A$ and $g: A^{m} \rightarrow A$ commute, and we write $f \perp g$, if

$$
\begin{aligned}
& g\left(f\left(a_{11}, \ldots, a_{1 n}\right), \ldots, f\left(a_{m 1}, \ldots, a_{m n}\right)\right) \\
& \quad=f\left(g\left(a_{11}, \ldots, a_{m 1}\right), \ldots, g\left(a_{1 n}, \ldots, a_{m n}\right)\right),
\end{aligned}
$$

for all $a_{11}, \ldots, a_{n m} \in A$. A self-commuting operation is one that commutes with itself. Clearly, every unary operation is self-commuting. An algebra $\mathbf{A}=(A ; F)$ is entropic if any two of its fundamental operations commute, i.e., for all $f, g \in F$, A satisfies the identity

$$
\begin{aligned}
& g\left(f\left(x_{11}, \ldots, x_{1 n}\right), \ldots, f\left(x_{m 1}, \ldots, x_{m n}\right)\right) \\
& \quad \approx f\left(g\left(x_{11}, \ldots, x_{m 1}\right), \ldots, g\left(x_{1 n}, \ldots, x_{m n}\right)\right) .
\end{aligned}
$$

Note that a groupoid $(A ; \cdot)$ is entropic if it satisfies the identity

$$
(x y) \cdot(z u) \approx(x z) \cdot(y u) .
$$

A weaker version of the entropic law is the so-called generalized entropic property. An algebra $\mathbf{A}=(A ; F)$ has the generalized entropic property if, for every $n$-ary $f \in F$ and every $m$-ary $g \in F$, there exist $m$-ary terms $t_{1}, \ldots, t_{n}$ of $\mathbf{A}$ such that $\mathbf{A}$ satisfies the identity

$$
\begin{aligned}
& g\left(f\left(x_{11}, \ldots, x_{n 1}\right), \ldots, f\left(x_{1 m}, \ldots, x_{n m}\right)\right) \\
& \quad \approx f\left(t_{1}\left(x_{11}, \ldots, x_{1 m}\right), \ldots, t_{n}\left(x_{n 1}, \ldots, x_{n m}\right)\right) .
\end{aligned}
$$

In particular, a groupoid $\mathbf{A}=(A ; \cdot)$ has the generalized entropic property if there are two binary terms $t_{1}$ and $t_{2}$ such that $\mathbf{A}$ satisfies the identity

$$
(x y) \cdot(z u) \approx t_{1}(x, z) \cdot t_{2}(y, u) .
$$

We say that an algebra $(A ; F)$ has the subalgebras property if, for each $n$-ary operation $f \in F$, the complex product

$$
f\left(A_{1}, \ldots, A_{n}\right):=\left\{f\left(a_{1}, \ldots, a_{n}\right) \mid a_{1} \in A_{1}, \ldots, a_{n} \in A_{n}\right\}
$$

of its (non-empty) subalgebras $A_{1}, \ldots, A_{n}$ is again a subalgebra.

It was shown in [1] that if $\mathcal{V}$ is an arbitrary variety of algebras, then each algebra in $\mathcal{V}$ has the subalgebras property if and only if each algebra in $\mathcal{V}$ has the generalized entropic property. (The result for a variety of groupoids was earlier proved by T. Evans [6].)

Some relationships between entropy and generalized entropy were investigated in [1]. For example, it was shown there that if an idempotent semigroup (a band) has the generalized entropic property, then it is entropic. For further information on entropic algebras in general and idempotent entropic algebras (modes) in particular, see [11]. 
This paper is devoted to algebras $(A ; F)$ with a neutral element or with a semigroup reduct $(A ; f)$. We investigate relationships between the generalized entropic property and the commutativity of the fundamental operations of $(A ; F)$. (In aggregation theory, the entropic laws for each $f \in F$ and associative binary operation - are called the generalized Cauchy equations (see [8, Sect. 2.5.2]).) In general, the generalized entropic property and commutativity are not equivalent, but in some cases they are.

The paper is organized as follows. The role of a neutral element is described in Sect. 2. In particular, we generalize the Eckmann-Hilton Theorem for $n$-ary operations and we prove that an algebra with a neutral element has the generalized entropic property if and only if it is derived from a commutative monoid. In Sect. 3, we investigate some expanded semigroups, and we show that for inverse semigroups, the generalized entropic property is also equivalent to commutativity. Finally, in Sect. 4, we survey some known results about the (generalized) entropy of $n$-semigroups.

\section{Algebras with a neutral element}

It is clear that a commutative semigroup is entropic. It is also easy to find an example of a non-commutative semigroup which is entropic. The simplest one is any left-zero band $(A ; \cdot)$ (a groupoid with $x y \approx x$ ). Moreover, it was shown in [1] that the variety of groupoids satisfying the identity

$$
\left(x_{1} x_{2}\right)\left(x_{3} x_{4}\right) \approx\left(x_{3} x_{1}\right)\left(x_{2} x_{4}\right)
$$

has the generalized entropic property, but it is not entropic. In particular, there exist non-commutative semigroups that have the generalized entropic property but are not entropic. But when we "add" to a semigroup a neutral element the situation changes: entropy and generalized entropy are known to be equivalent for algebras with a neutral element (see Theorem 2.1 below).

An element $e \in A$ is said to be neutral for an operation $f: A^{n} \rightarrow A$ if

$$
f(a, e, \ldots, e)=f(e, a, e, \ldots, e)=\cdots=f(e, \ldots, e, a)=a
$$

for every $a \in A$. Note that every element of $A$ is neutral for the identity map on $A$; this is the only unary operation that has a neutral element. Nullary operations do not have neutral elements. We say that $e$ is neutral for an algebra $(A ; F)$ if it is neutral for each operation $f \in F$. Observe that if $e$ is neutral for an algebra $(A ; F)$, then $\{e\}$ is a subalgebra of $(A ; F)$.

Theorem $2.1([1])$ Let $(A ; F)$ be an algebra with a neutral element. Then, $(A ; F)$ has the generalized entropic property if and only if it is entropic.

In particular, every groupoid $(A ; \cdot)$ with a neutral element $e$ has the generalized entropic property if and only if it is entropic.

Eckmann and Hilton [5] established the following strong result about commuting binary operations with a common neutral element. 
Theorem 2.2 ([5]) Assume that $f$ and $g$ are binary operations on $A$ and $e \in A$ is neutral for both $f$ and $g$. If $f \perp g$, then $f=g$ and $f$ and $g$ are commutative. Consequently, $f$ and $g$ are associative.

By Theorem 2.2, every entropic groupoid $(A ; \cdot)$ with a neutral element is a commutative monoid. Hence, a monoid has the generalized entropic property if and only if it is commutative. Moreover, by the results of [6] (see also [1]), a group has the generalized entropic property if and only if it is commutative.

In the remainder of this section, we are going to find an explicit description of algebras with a neutral element that have the generalized entropic property (or, equivalently, are entropic). First we will generalize the Eckmann-Hilton theorem for $n$-ary operations, assuming a slightly weaker form of neutral elements. Let $n \geq 2$. We say that an $(n-1)$-tuple $\left(e_{1}, \ldots, e_{n-1}\right) \in A^{n-1}$ is neutral for an operation $f: A^{n} \rightarrow A$ if

$$
\begin{aligned}
f\left(a, e_{\sigma(1)}, \ldots, e_{\sigma(n-1)}\right) & =\cdots=f\left(e_{\sigma(1)}, \ldots, e_{\sigma(r)}, a, e_{\sigma(r+1)}, \ldots, e_{\sigma(n-1)}\right) \\
& =\cdots=f\left(e_{\sigma(1)}, \ldots, e_{\sigma(n-1)}, a\right)=a
\end{aligned}
$$

for every $a \in A$ and any permutation $\sigma \in S_{n-1}$. Note that an element $e$ is neutral for $f: A^{n} \rightarrow A$ if and only if the $(n-1)$-tuple $(e, \ldots, e)$ is neutral for $f$.

An operation $f$ is totally symmetric if for all permutations $\sigma$ of $\{1, \ldots, n\}$, we have $f\left(a_{1}, \ldots, a_{n}\right)=f\left(a_{\sigma(1)}, \ldots, a_{\sigma(n)}\right)$ for all $a_{1}, \ldots, a_{n} \in A$.

Lemma 2.3 Let $f$ and $g$ be $n$-ary operations on $A$, and assume that $\left(e_{1}, \ldots, e_{n-1}\right) \in$ $A^{n-1}$ is neutral for both $f$ and $g$. If $f \perp g$, then $f=g$ and $f$ and $g$ are totally symmetric.

Before entering into the proof, let us recall that a Latin square of order $n$ is an $n \times n$ matrix with entries from the set $\{1, \ldots, n\}$ such that each element of $\{1, \ldots, n\}$ occurs exactly once in each row and exactly once in each column. There exist Latin squares of every order $n \geq 1$. If we permute the rows or the columns of a Latin square, the resulting matrix is again a Latin square. Based on this observation, it is easy to see (and well known) that for every permutation $\sigma$ of $\{1, \ldots, n\}$ and every element $k \in\{1, \ldots, n\}$, there exists a Latin square of order $n$ such that, for every $j \in\{1, \ldots, n\}$, the entry in row $\sigma(j)$, column $j$ equals $k$.

Proof of Lemma 2.3 If $n=1$, then our definitions yield that $f$ and $g$ are both equal to the identity map on $A$, and in this case the claim clearly holds. We may thus assume that $n \geq 2$. Let $\sigma$ be an arbitrary permutation of $\{1, \ldots, n\}$. Let $M=\left(m_{i j}\right)$ be a Latin square of order $n$ such that $m_{\sigma(j) j}=n$ for every $j \in\{1, \ldots, n\}$. Let $a_{1}, \ldots, a_{n}$ be arbitrary elements of $A$, and consider Eq. (1.1) for

$$
a_{i j}=\left\{\begin{array}{ll}
a_{i}, & \text { if } i=\sigma(j), \\
e_{m_{i j}}, & \text { if } i \neq \sigma(j),
\end{array} \quad(1 \leq i \leq n, 1 \leq j \leq n)\right.
$$


The left-hand side of (1.1) yields

$$
\begin{aligned}
& g\left(f\left(e_{\rho_{1}(1)}, \ldots, a_{1}, \ldots, e_{\rho_{1}(n-1)}\right), \ldots, f\left(e_{\rho_{n}(1)}, \ldots, a_{n}, \ldots, e_{\rho_{n}(n-1)}\right)\right) \\
& \quad=g\left(a_{1}, \ldots, a_{n}\right),
\end{aligned}
$$

for some permutations $\rho_{1}, \ldots, \rho_{n}$ of $\{1, \ldots, n-1\}$, and the right-hand side yields

$$
\begin{aligned}
& f\left(g\left(e_{\kappa_{1}(1)}, \ldots, a_{\sigma(1)}, \ldots, e_{\kappa_{1}(n-1)}\right), \ldots, g\left(e_{\kappa_{n}(1)}, \ldots, a_{\sigma(n)}, \ldots, e_{\kappa_{n}(n-1)}\right)\right) \\
& \quad=f\left(a_{\sigma(1)}, \ldots, a_{\sigma(n)}\right),
\end{aligned}
$$

for some permutations $\kappa_{1}, \ldots, \kappa_{n}$ of $\{1, \ldots, n-1\}$. These are equal by the assumption that $f \perp g$. Taking $\sigma$ to be the identity permutation, we have that $f=g$. Letting $\sigma$ vary over the symmetric group $S_{n}$, we can conclude that $f$ is totally symmetric.

Consequently, if $f$ has a neutral element and $f$ is self-commuting, then $f$ is totally symmetric.

The notion of associativity can be generalized to $n$-ary operations as follows. An $n$-ary operation $f: A^{n} \rightarrow A$ is said to be associative if the following associative laws hold:

$$
\begin{aligned}
f( & \left.f\left(a_{1}, \ldots, a_{n}\right), a_{n+1}, \ldots, a_{2 n-1}\right) \\
& =\cdots=f\left(a_{1}, \ldots, a_{r}, f\left(a_{r+1}, \ldots, a_{r+n}\right), a_{r+n+1}, \ldots, a_{2 n-1}\right) \\
& =\cdots=f\left(a_{1}, \ldots, a_{n-1}, f\left(a_{n}, \ldots, a_{2 n-1}\right)\right),
\end{aligned}
$$

for all $a_{1}, \ldots, a_{2 n-1} \in A$. An algebra $(A ; f)$ with one $n$-ary associative operation $f$ is called an $n$-semigroup.

Lemma 2.4 If $f: A^{n} \rightarrow A$ has a neutral $(n-1)$-tuple and $f \perp f$, then $f$ is associative.

Proof First we will show that for any $a_{1}, \ldots, a_{n}, a_{n+1}, \ldots, a_{2 n-1} \in A$

$$
\begin{aligned}
& f\left(f\left(a_{1}, \ldots, a_{n-1}, a_{n}\right), a_{n+1}, a_{n+2}, \ldots, a_{2 n-1}\right) \\
& \quad=f\left(f\left(a_{1}, \ldots, a_{n-1}, a_{n+1}\right), a_{n}, a_{n+2}, \ldots, a_{2 n-1}\right) .
\end{aligned}
$$

Let $\left(e_{1}, \ldots, e_{n-1}\right)$ be a neutral $(n-1)$-tuple for $f$ and let $\pi \in S_{n-1}$ be any cycle of length $n-1$. By Lemma 2.3, $f$ is totally symmetric. Hence we have

$$
\begin{aligned}
f( & \left.f\left(a_{1}, \ldots, a_{n}\right), a_{n+1}, \ldots, a_{2 n-1}\right) \\
= & f\left(f\left(a_{1}, \ldots, a_{n-1}, a_{n}\right),\right. \\
& \left.\quad f\left(e_{\pi^{1}(1)}, \ldots, e_{\pi^{1}(n-1)}, a_{n+1}\right), \ldots, f\left(e_{\pi^{n-1}(1)}, \ldots, e_{\pi^{n-1}(n-1)}, a_{2 n-1}\right)\right) \\
= & f\left(f\left(a_{1}, e_{\pi^{1}(1)}, \ldots, e_{\pi^{n-1}(1)}\right), \ldots, f\left(a_{n-1}, e_{\pi^{1}(n-1)}, \ldots, e_{\pi^{n-1}(n-1)}\right),\right. \\
& \left.\quad f\left(a_{n}, a_{n+1}, \ldots, a_{2 n-1}\right)\right)
\end{aligned}
$$




$$
\begin{aligned}
= & f\left(f\left(a_{1}, e_{\pi^{1}(1)}, \ldots, e_{\pi^{n-1}(1)}\right), \ldots, f\left(a_{n-1}, e_{\pi^{1}(n-1)}, \ldots, e_{\pi^{n-1}(n-1)}\right),\right. \\
& \left.\quad f\left(a_{n+1}, a_{n}, a_{n+2}, \ldots, a_{2 n-1}\right)\right) \\
= & f\left(f\left(a_{1}, \ldots, a_{n-1}, a_{n+1}\right), f\left(e_{\pi^{1}(1)}, \ldots, e_{\pi^{1}(n-1)}, a_{n}\right),\right. \\
& \left.\quad f\left(e_{\pi^{2}(1)}, \ldots, e_{\pi^{2}(n-1)}, a_{n+2}\right), \ldots, f\left(e_{\pi^{n-1}(1)}, \ldots, e_{\pi^{n-1}(n-1)}, a_{2 n-1}\right)\right) \\
= & f\left(f\left(a_{1}, \ldots, a_{n-1}, a_{n+1}\right), a_{n}, a_{n+2}, \ldots, a_{2 n-1}\right),
\end{aligned}
$$

where the first and the fifth equalities hold because $\left(e_{1}, \ldots, e_{n-1}\right)$ is neutral, the second and the fourth equalities hold by the assumption that $f \perp f$, and the third equality holds by the total symmetry of $f$. Now, it is easy to deduce from (2.2) and the total symmetry of $f$ that $f$ is associative.

The following theorem generalizes the Eckmann-Hilton theorem for $n$-ary operations.

Theorem 2.5 If $f$ and $g$ are $n$-ary operations on $A, n \geq 2,\left(e_{1}, \ldots, e_{n-1}\right) \in A^{n-1}$ is neutral for both $f$ and $g$, and $f \perp g$, then $f=g$ and $f$ and $g$ are totally symmetric and associative.

Proof Follows immediately from Lemmas 2.3 and 2.4.

Corollary 2.6 Each self-commuting operation with a neutral tuple (or with a neutral element) $i$ s associative and totally symmetric.

Lemma 2.7 Let $(A ; F)$ be an algebra with one-element subalgebras $\left\{e_{1}\right\}, \ldots,\left\{e_{n-1}\right\}$, and assume that the $(n-1)$-tuple $\left(e_{1}, \ldots, e_{n-1}\right)$ is neutral for an n-ary operation $f \in F$. If $(A ; F)$ has the generalized entropic property, then $f$ commutes with each operation $g \in F$.

Proof Let $g: A^{m} \rightarrow A$ be an operation in $F$. By the generalized entropic property, there exist terms $t_{1}, \ldots, t_{n}$ such that $(A ; F)$ satisfies the identity (1.3). Hence, for every $k=1, \ldots, n$,

$$
\begin{aligned}
g( & \left.x_{1}, \ldots, x_{m}\right) \\
& \approx g\left(f\left(e_{1}, \ldots, e_{k-1}, x_{1}, e_{k}, \ldots, e_{n-1}\right), \ldots, f\left(e_{1}, \ldots, e_{k-1}, x_{m}, e_{k}, \ldots, e_{n-1}\right)\right) \\
& \approx f\left(t_{1}\left(e_{1}, \ldots, e_{1}\right), \ldots, t_{k}\left(x_{1}, \ldots, x_{m}\right), \ldots, t_{n}\left(e_{n-1}, \ldots, e_{n-1}\right)\right) \\
& \approx f\left(e_{1}, \ldots, t_{k}\left(x_{1}, \ldots, x_{m}\right), \ldots, e_{n-1}\right) \approx t_{k}\left(x_{1}, \ldots, x_{m}\right) .
\end{aligned}
$$

This implies that

$$
\begin{aligned}
& g\left(f\left(x_{11}, \ldots, x_{1 n}\right), \ldots, f\left(x_{m 1}, \ldots, x_{m n}\right)\right) \\
& \quad \approx f\left(g\left(x_{11}, \ldots, x_{m 1}\right), \ldots, g\left(x_{1 n}, \ldots, x_{m n}\right)\right),
\end{aligned}
$$

that is, $f \perp g$. 
As a by-product of the results we have obtained so far, we can give a proof of Theorem 2.1.

Proof of Theorem 2.1 Assume that $(A ; F)$ has the generalized entropic property, and let $e$ be a neutral element of $(A ; F)$. Since $e$ is neutral for every operation in $F$, it follows from Lemma 2.7 that $f \perp g$ for all $f, g \in F$, i.e., $(A ; F)$ is entropic. The converse implication is clear.

Let $f: A^{n} \rightarrow A$ be an arbitrary operation of arity $n \geq 1$. For $\ell \geq 0$, we define the operation $f^{(\ell)}$ of arity $N(\ell):=\ell(n-1)+1$ recursively as follows: $f^{(0)}:=\mathrm{id}_{A}$, and for $\ell \geq 0$, we let

$$
f^{(\ell+1)}\left(a_{1}, \ldots, a_{N(\ell+1)}\right)=f\left(f^{(\ell)}\left(a_{1}, \ldots, a_{N(\ell)}\right), a_{N(\ell)+1}, \ldots, a_{N(\ell+1)}\right),
$$

for all $a_{1}, \ldots, a_{N(\ell+1)} \in A$. Note that $f^{(1)}=f$. (Note also that if $*$ denotes the binary composition as in iterative algebras [9], we have that $f^{(\ell+1)}=f * f^{(\ell)}$ for all $\ell \geq 0$.) We say that an algebra $\left(A ;\left(f_{i}\right)_{i \in I}\right)$ of type $\tau=\left(n_{i}\right)_{i \in I}$ is the $\tau$-algebra derived from $f$ if for every $i \in I$ there exists an integer $\ell_{i} \geq 0$ such that $n_{i}=N\left(\ell_{i}\right)$ and $f_{i}=f^{\left(\ell_{i}\right)}$.

Our main result concerning entropy and the generalized entropic property in algebras with a neutral element is the following.

Theorem 2.8 Let $\mathbf{A}=(A ; F)$ be an algebra of type $\tau$ with a neutral element $e$. Then $\mathbf{A}$ is entropic (or, equivalently, $\mathbf{A}$ has the generalized entropic property) if and only if there exists a commutative monoid $(A ; f, e)$ such that $\mathbf{A}$ is the $\tau$-algebra derived from $f$.

In order to prove Theorem 2.8, we will first establish a few lemmas. Our first lemma was proved by Couceiro and Marichal [2, Proposition 2.5].

Lemma 2.9 ([2]) Let $f: A^{n} \rightarrow A$ be an associative operation.

(i) For all $\ell, m \geq 0$ and for every $i=0, \ldots, N(\ell)$, the equality

$$
\begin{aligned}
& f^{(\ell)}\left(a_{1}, \ldots, a_{i}, f^{(m)}\left(a_{i+1}, \ldots, a_{i+N(m)}\right), a_{i+N(m)+1}, \ldots, a_{N(\ell+m)}\right) \\
& \quad=f^{(\ell+m)}\left(a_{1}, \ldots, a_{N(\ell+m)}\right)
\end{aligned}
$$

holds for all $a_{1}, \ldots, a_{N(\ell+m)} \in A$.

(ii) For all $\ell \geq 0, m_{1}, \ldots, m_{N(\ell)} \geq 0$, the equality

$$
f^{(\ell)}\left(f^{\left(m_{1}\right)}\left(\mathbf{a}_{1}\right), \ldots, f^{\left(m_{N(\ell)}\right)}\left(\mathbf{a}_{N(\ell)}\right)\right)=f^{\left(\ell+\sum_{i=1}^{N(\ell)} m_{i}\right)}\left(\mathbf{a}_{1}, \ldots, \mathbf{a}_{N(\ell)}\right)
$$

holds for all $\mathbf{a}_{i} \in A^{N\left(m_{i}\right)}(1 \leq i \leq N(\ell))$.

Lemma 2.10 Let $f: A^{n} \rightarrow A$ be associative and totally symmetric. Then for every $\ell \geq 0$, the operation $f^{(\ell)}$ is totally symmetric. 
Proof The claim clearly holds if $n=1$, so we assume that $n \geq 2$. Since the permutations $(1,2)$ and $(1,2, \ldots, N(\ell))$ generate the symmetric group $S_{N(\ell)}$, it is sufficient to show that

$$
\begin{aligned}
& f^{(\ell)}\left(a_{1}, \ldots, a_{N(\ell)}\right)=f^{(\ell)}\left(a_{2}, a_{1}, a_{3}, a_{4}, \ldots, a_{N(\ell)}\right) \quad \text { and } \\
& f^{(\ell)}\left(a_{1}, \ldots, a_{N(\ell)}\right)=f^{(\ell)}\left(a_{N(\ell)}, a_{1}, a_{2}, \ldots, a_{N(\ell)-1}\right)
\end{aligned}
$$

hold for all $a_{1}, \ldots, a_{N(\ell)} \in A$.

For the first equality, we have

$$
\begin{aligned}
f^{(\ell)}\left(a_{1}, \ldots, a_{N(\ell)}\right) & =f^{(\ell-1)}\left(f^{(1)}\left(a_{1}, \ldots, a_{n}\right), a_{n+1}, \ldots, a_{N(\ell)}\right) \\
& =f^{(\ell-1)}\left(f^{(1)}\left(a_{2}, a_{1}, a_{3}, a_{4}, \ldots, a_{n}\right), a_{n+1}, \ldots, a_{N(\ell)}\right) \\
& =f^{(\ell)}\left(a_{2}, a_{1}, a_{3}, a_{4}, \ldots, a_{N(\ell)}\right),
\end{aligned}
$$

where the first and the third equalities hold by Lemma 2.9(i), and the second equality holds by the total symmetry of $f=f^{(1)}$.

For the second equality, we have

$$
\begin{aligned}
f^{(\ell)}\left(a_{1}, \ldots, a_{N(\ell)}\right) & =f^{(1)}\left(f^{(\ell-1)}\left(a_{1}, \ldots, a_{N(\ell-1)}\right), a_{N(\ell-1)+1}, \ldots, a_{N(\ell)}\right) \\
& =f^{(1)}\left(a_{N(\ell)}, f^{(\ell-1)}\left(a_{1}, \ldots, a_{N(\ell-1)}\right), a_{N(\ell-1)+1}, \ldots, a_{N(\ell)-1}\right) \\
& =f^{(\ell)}\left(a_{N(\ell)}, a_{1}, a_{2}, \ldots, a_{N(\ell)-1}\right),
\end{aligned}
$$

where the first and the third equalities hold by Lemma 2.9(i), and the second equality holds by the total symmetry of $f=f^{(1)}$.

Lemma 2.11 Let $f: A^{n} \rightarrow A$ be associative and totally symmetric. Then for every $\ell, m \geq 0$, the operations $f^{(\ell)}$ and $f^{(m)}$ commute.

Proof We need to show that $f^{(\ell)} \perp f^{(m)}$ for all $\ell, m \geq 0$, i.e.,

$$
\begin{aligned}
& f^{(\ell)}\left(f^{(m)}\left(a_{1,1}, \ldots, a_{1, N(m)}\right), \ldots, f^{(m)}\left(a_{N(\ell), 1}, \ldots, a_{N(\ell), N(m)}\right)\right) \\
& =f^{(m)}\left(f^{(\ell)}\left(a_{1,1}, \ldots, a_{N(\ell), 1}\right), \ldots, f^{(\ell)}\left(a_{1, N(m)}, \ldots, a_{N(\ell), N(m)}\right)\right)
\end{aligned}
$$

holds for all $a_{i, j} \in A, 1 \leq i \leq N(\ell), 1 \leq j \leq N(m)$. By Lemma 2.9(ii), the left-hand side of (2.3) equals

$$
f^{(\ell+N(\ell) m)}\left(a_{1,1}, \ldots, a_{1, N(m)}, \ldots, a_{N(\ell), 1}, \ldots, a_{N(\ell), N(m)}\right),
$$

while the right-hand side equals

$$
f^{(m+N(m) \ell)}\left(a_{1,1}, \ldots, a_{N(\ell), 1}, \ldots, a_{1, N(m)}, \ldots, a_{N(\ell), N(m)}\right) .
$$

Observe that

$$
\ell+N(\ell) m=\ell m(n-1)+\ell+m=m+N(m) \ell
$$


and

$$
\begin{aligned}
N(\ell+N(\ell) m) & =N(m+N(m) \ell)=N(\ell) N(m) \\
& =\ell m(n-1)^{2}+(\ell+m)(n-1)+1,
\end{aligned}
$$

so (2.4) and (2.5) do indeed refer to the same function. Lemma 2.10, together with our assumption that $f$ is totally symmetric, implies that $f^{(\ell m(n-1)+\ell+m)}$ is totally symmetric as well; hence (2.4) and (2.5) are equal, and the proof is complete.

Corollary 2.12 Each associative and totally symmetric operation is self-commuting.

Lemma 2.13 Let $f: A^{n} \rightarrow A$ be associative, and let $g: A^{\ell(n-1)+1} \rightarrow A$ for some $\ell \geq 1$. Assume that $e \in A$ is neutral for both $f$ and $g$. If $f \perp g$, then $g=f^{(\ell)}$.

Proof We will prove by induction that for every $k \in\{0, \ldots, \ell\}$, the equality

$$
g(a_{1}, \ldots, a_{k(n-1)+1}, \underbrace{e, \ldots, e}_{(\ell-k)(n-1)})=f^{(k)}\left(a_{1}, \ldots, a_{k(n-1)+1}\right)
$$

holds for all $a_{1}, \ldots, a_{k(n-1)+1} \in A$. This shows (for $k=\ell$ ) that $g=f^{(\ell)}$.

Since $e$ is neutral for $g$, we have that

$$
g\left(a_{1}, e, \ldots, e\right)=a_{1}=f^{(0)}\left(a_{1}\right),
$$

and equality (2.6) holds for $k=0$. Assume then that (2.6) holds for $k=r \geq 0$. We have

$$
\begin{aligned}
g\left(a_{1}, \ldots, a_{(r+1)(n-1)+1}, e, \ldots, e\right) & \\
= & g\left(f\left(a_{1}, e, \ldots, e\right), \ldots, f\left(a_{r(n-1)+1}, e, \ldots, e\right),\right. \\
& \quad f\left(e, a_{r(n-1)+2}, e, \ldots, e\right), \ldots, f\left(e, \ldots, e, a_{(r+1)(n-1)+1}\right), \\
& \quad f(e, \ldots, e), \ldots, f(e, \ldots e)) \\
= & f\left(g\left(a_{1}, \ldots, a_{r(n-1)+1}, e, \ldots, e\right),\right. \\
& \left.\quad g\left(e, \ldots, e, a_{r(n-1)+2}, e, \ldots, e\right), \ldots, g\left(e, \ldots, e, a_{(r+1)(n-1)+1}, e, \ldots, e\right)\right) \\
= & f\left(f^{(r)}\left(a_{1}, \ldots, a_{r(n-1)+1}\right), a_{r(n-1)+2}, \ldots, a_{(r+1)(n-1)+1}\right) \\
= & f^{(r+1)}\left(a_{1}, \ldots, a_{(r+1)(n-1)+1}\right),
\end{aligned}
$$

where the first equality holds because $e$ is neutral for $f$, the second equality holds since $f \perp g$, the third equality holds by the induction hypothesis and by the assumption that $e$ is neutral for $f$, and the last equality holds by the definition of $f^{(r+1)}$. This shows that equality (2.6) holds also for $k=r+1$, and the inductive proof is complete. 
Proof of Theorem 2.8 By Theorem 2.1, entropy and generalized entropy are equivalent in algebras with a neutral element, so we may assume that $\mathbf{A}$ is entropic. Let $g \in F$ be an operation of arity $m \geq 2$, and define a binary operation $f$ by $f(a, b):=g(a, b, e, \ldots, e)$ for all $a, b \in A$. Since $g$ is associative and totally symmetric by Theorem 2.5 and $e$ is neutral for $g$, it is easy to verify that $(A ; f, e)$ is a commutative monoid. Furthermore, if $g$ commutes with an operation $h$ satisfying $h(e, \ldots, e)=e$, then also $f$ commutes with $h$. Thus, if $h$ is an $n$-ary operation in $F, n \geq 2$, then we have that $f \perp h$, and Lemma 2.13 implies that $h=f^{(n-1)}$. Furthermore, every unary operation in $F$ equals $\mathrm{id}_{A}=f^{(0)}$, and there are no nullary operations in $F$. We conclude that $\mathbf{A}$ is the $\tau$-algebra derived from $f$.

The converse implication is established in Lemma 2.11.

\section{Inverse semigroups and other expanded semigroups}

We now consider the problem of determining conditions when an expanded semigroup (an algebra $(A ; F)$ such that $F$ contains a binary associative operation) has the generalized entropic property. While a complete characterization eludes us, we investigate a few interesting special cases. Of course, we will assume that the algebra does not have a neutral element-otherwise, we know the answer from Theorem 2.8.

Let us start with a monoid $(A ;+, 0, \cdot)$ with one additional binary multiplication. Assume that $\{0\}$ is a subalgebra of $(A ; \cdot)$. Then by Lemma 2.7 , if $(A ;+, 0, \cdot)$ has the generalized entropic property, then + and $\cdot$ commute:

$$
(x+y)(z+u) \approx(x z)+(y u) .
$$

Hence, if $(A ;+, 0, \cdot)$ is a semiring with absorbing zero 0 , then

$$
x y \approx(x+0)(0+y) \approx(x 0)+(0 y) \approx 0 .
$$

Thus, we have obtained the following result.

Theorem 3.1 The only semirings with absorbing zero and with the generalized entropic property are zero-semirings.

In particular, if a ring $(A ;+, 0, \cdot)$ with a multiplicative neutral element 1 has the generalized entropic property, then $(A ;+, 0, \cdot)$ is trivial $(0=1)$. Moreover, Theorem 3.1 shows that there is no field which has the generalized entropic property.

Theorem 3.2 The only lattices with the generalized entropic property are the oneelement lattices.

Proof Assume that $\mathbf{L}=(A ; \wedge, \vee)$ is a lattice with the generalized entropic property. It is easy to see that any binary term operation $t(x, y)$ on $\mathbf{L}$ equals one of the following: $x, y, x \vee y, x \wedge y$. Straightforward calculations show that for every choice of binary terms $t_{1}$ and $t_{2}$, identity (1.3) for $f=\vee, g=\wedge$ holds only if $|A|=1$.

An inverse semigroup is an algebra $\mathbf{A}=\left(A ; \cdot,{ }^{-1}\right)$ of type $(2,1)$ that satisfies the following identities: 
$-x \cdot(y \cdot z) \approx(x \cdot y) \cdot z$ (associativity, i.e., $(A ; \cdot)$ is a semigroup),

$-x x^{-1} x \approx x$

$-\left(x^{-1}\right)^{-1} \approx x$,

$-(x y)^{-1} \approx y^{-1} x^{-1}$,

$-x x^{-1} x^{-1} x \approx x^{-1} x x x^{-1}$.

We say that an inverse semigroup $\mathbf{A}=\left(A ; \cdot,^{-1}\right)$ is commutative if $(A ; \cdot)$ is a commutative semigroup.

We will show that entropy, generalized entropy, and commutativity are equivalent properties in inverse semigroups. We first recall some notions and tools from the structure theory of inverse semigroups (see Petrich [10, Sects. II and XII]). Let $\rho$ be a congruence of a semigroup $S$. If $S / \rho$ is a semilattice, then $\rho$ is a semilattice congruence on $S$, and $S$ is referred to as a semilattice of semigroups $S_{\alpha}$, where the $S_{\alpha}$ are the $\rho$-classes. A Clifford semigroup is a semilattice of groups. A variety consisting solely of Clifford semigroups is a Clifford variety. The 5-element Brandt semigroup $B_{2}$ is the semigroup generated by $a$ and $b$, subject to the following relations:

$$
a^{2}=b^{2}=0, \quad a b a=a, \quad b a b=b .
$$

Note that $B_{2}$ is an inverse semigroup with $a^{-1}=b, b^{-1}=a,(a b)^{-1}=a b,(b a)^{-1}=$ $b a, 0^{-1}=0$.

Proposition 3.3 (see Petrich [10, Proposition XII.4.13]) A variety $\mathcal{V}$ of inverse semigroups is a Clifford variety if and only if $B_{2} \notin \mathcal{V}$.

From the description of the free monogenic inverse semigroup [10, Chap. XI] it follows that for every unary inverse semigroup term $t(x)$, there exist numbers $p, q$, $r$ with $0 \leq p \leq q, 0 \leq r \leq q, q>0$, such that the identity $t(x) \approx x^{-p} x^{q} x^{-r}$ holds in the variety of all inverse semigroups. (We make use of the convention that $x^{0}$ is an empty symbol.)

Theorem 3.4 For an inverse semigroup A, the following conditions are equivalent.

(i) A is entropic.

(ii) A has the generalized entropic property.

(iii) $\mathbf{A}$ is commutative.

Proof (i) $\Longrightarrow$ (ii) Entropy implies generalized entropy.

(ii) $\Longrightarrow$ (iii) Let $\mathbf{A}$ be an inverse semigroup, and assume that $\mathbf{A}$ has the generalized entropic property. Then there exist unary terms $t_{1}$ and $t_{2}$ of $\mathbf{A}$ such that $\mathbf{A}$ satisfies the identity

$$
(x y)^{-1} \approx t_{1}(x) \cdot t_{2}(y) .
$$

We may assume that $t_{1}(x)=x^{-p_{1}} x^{q_{1}} x^{-r_{1}}$ and $t_{2}(y)=y^{-p_{2}} y^{q_{2}} y^{-r_{2}}$ for some $p_{1}, q_{1}, r_{1}, p_{2}, q_{2}, r_{2}$ satisfying $0 \leq p_{i} \leq q_{i}, 0 \leq r_{i} \leq q_{i}, q_{i}>0$ for $i \in\{1,2\}$. Identity (3.1) fails in the 5-element Brandt semigroup $B_{2}$. (We leave the verification of the details to the reader: if $t_{1}(x)=x$ and $t_{2}(y)=y$ or $t_{1}(x)=x x^{-1}$ and $t_{2}(y)=y^{-1} y$, then evaluate $x$ and $y$ at $a b$ and $a$, respectively; otherwise evaluate $x$ and $y$ at $a$ and $b$, 
respectively.) By Proposition 3.3, A is a semilattice of groups. Every group satisfying (3.1) is abelian. (To see this, observe that for a group $\mathbf{G}=\left(G ; \cdot,{ }^{-1}\right)$, identity (3.1) means that there exist integers $m$ and $n$ such that $\mathbf{G}$ satisfies $x y \approx y^{m} x^{n}$. Here $x^{0}$ stands for the neutral element $e$ of $(G ; \cdot)$. This implies that $x \approx x e \approx e^{m} x^{n} \approx x^{n}$ and $y \approx e y \approx y^{m} e^{n} \approx y^{m}$. Consequently, $x y \approx y x$.) Hence $\mathbf{A}$ is a semilattice of abelian groups, and so $\mathbf{A}$ is commutative.

(iii) $\Longrightarrow$ (i) Assume that $\mathbf{A}$ is commutative. Then $(x y)^{-1} \approx(y x)^{-1} \approx x^{-1} y^{-1}$, and Eq. (1.2) holds for $g={ }^{-1}$ and $f=\cdot$. Equation (1.2) holds trivially for $g=$ $f={ }^{-1}$, because every unary operation is self-commuting. Because each commutative semigroup is entropic, Eq. (1.2) also holds for $g=f=\cdot$. Thus, $\mathbf{A}$ is entropic.

\section{4 n-Semigroups}

The current study gives rise in an obvious way to the following interesting problem: find necessary and sufficient conditions for an $n$-semigroup to be entropic (or to have the generalized entropic property). To the best of our knowledge, this is an open problem. We will survey here some known results when restricted to some special classes of $n$-semigroups.

Theorems 3.4 and 4.3 show that in inverse semigroups or in $n$-semigroups with a neutral element, entropy (or, equivalently, generalized entropy) is equivalent to commutativity (or total symmetry).

An $n$-semigroup $(A ; f)$ is called an $n$-group if for every $i \in\{1, \ldots, n\}$ and for all $a_{1}, \ldots, a_{i-1}, a_{i+1}, \ldots, a_{n}, c \in A$, there exists a unique $b \in A$ such that $f\left(a_{1}, \ldots, a_{i-1}, b, a_{i+1}, \ldots, a_{n}\right)=c$. A group is a 2-group in this sense. We will refer to $n$-semigroups with a neutral element as $n$-monoids, and to $n$-semigroups with a neutral $(n-1)$-tuple as generalized $n$-monoids. A monoid is a 2 -monoid and a generalized 2-monoid in this sense.

Let $1 \leq i<j \leq n$. An operation $f: A^{n} \rightarrow A$ is said to be $(i, j)$-commutative if

$f\left(a_{1}, \ldots, a_{i}, \ldots, a_{j}, \ldots, a_{n}\right)=f\left(a_{1}, \ldots, a_{i-1}, a_{j}, a_{i+1}, \ldots, a_{j-1}, a_{i}, a_{j+1}, \ldots, a_{n}\right)$

for all $a_{1}, \ldots, a_{n} \in A$. In particular, a $(1, n)$-commutative operation is called semiabelian.

As we have already noticed a binary associative and commutative operation is entropic. This observation is also true for a more general case. Dörnte [3] (see also [7]) proved that any $n$-ary semiabelian and associative operation is self-commuting. (Dörnte stated this result for $n$-groups, but his proof works for $n$-semigroups.)

\section{Theorem 4.1 ([3]) Each semiabelian n-semigroup is entropic.}

On the other hand Głazek and Gleichgewicht showed in [7] that an $n$-group is semiabelian if and only if it is entropic. As a consequence of Theorems 2.5 and 4.1, a similar result is also true for $n$-monoids.

Corollary 4.2 An n-monoid is semiabelian if and only if it is entropic. 
In fact we have even more.

Theorem 4.3 Let $(A ; f)$ be an algebra with one $n$-ary operation that has a neutral $(n-1)$-tuple. Then $(A ; f)$ is entropic (or equivalently has generalized entropic property) if and only if it is semiabelian generalized n-monoid.

From Theorem 5 in [4] we can conclude that a generalized $n$-monoid is $(i, i+1)$ commutative for $1 \leq i \leq n-1$ if and only if it is totally symmetric. Note that by Theorems 2.5 and 4.1 any generalized $n$-monoid is totally symmetric if and only if it is semiabelian.

Moreover, Dudek formulated another equivalent condition for semiabelianness of some $n$-semigroups.

Theorem 4.4 ([4]) Let $(A ; f)$ be an $n$-semigroup in which there exist $a_{1}, \ldots$, $a_{n-1} \in A$ such that for all $a \in A, f\left(a_{1}, \ldots, a_{n-1}, a\right)=a$ or $f\left(a, a_{1}, \ldots, a_{n-1}\right)=a$. Then, $(A ; f)$ is semiabelian if and only if $f\left(a, a_{1}, \ldots, a_{n-2}, b\right)=f\left(b, a_{1}, \ldots\right.$, $\left.a_{n-2}, a\right)$ for all $a, b \in A$.

Hence, by Corollary 4.2 we immediately have.

Corollary 4.5 An n-monoid $(A ; f, e)$ is entropic if and only if $f(a, e, \ldots, e, b)=$ $f(b, e, \ldots, e, a)$ for all $a, b \in A$.

Acknowledgements The authors are indebted to the anonymous referee for suggesting the proof of Theorem 3.4 and for other constructive remarks that helped improve the manuscript.

Open Access This article is distributed under the terms of the Creative Commons Attribution License which permits any use, distribution, and reproduction in any medium, provided the original author(s) and the source are credited.

\section{References}

1. Adaricheva, K., Pilitowska, A., Stanovský, D.: Complex algebras of subalgebras. Algebra Logika 47, 655-686 (2008) (in Russian). English translation: Algebra Logic 47, 367-383 (2008)

2. Couceiro, M., Marichal, J.-L.: Aczélian n-ary semigroups. Semigroup Forum 85, 81-90 (2012)

3. Dörnte, W.: Untersuchungen über einen verallgemeinerten Gruppenbegriff. Math. Z. 29, 1-19 (1928)

4. Dudek, W.A.: Remarks on $n$-groups. Demonstr. Math. 13(1), 165-181 (1980)

5. Eckmann, B., Hilton, P.J.: Group-like structures in general categories I-multiplications and comultiplications. Math. Ann. 145, 227-255 (1962)

6. Evans, T.: Properties of algebras almost equivalent to identities. J. Lond. Math. Soc. 35, 53-59 (1962)

7. Głazek, K., Gleichgewicht, B.: Abelian n-groups. In: Csákány, B., Fried, E., Schmidt, E.T. (eds.) Universal Algebra. Colloquia Mathematica Societatis János Bolyai, vol. 29, pp. 321-329. North-Holland, Amsterdam (1982)

8. Grabisch, M., Marichal, J.-L., Mesiar, R., Pap, E.: Aggregation Functions. Encyclopedia of Mathematics and Its Applications, vol. 127. Cambridge University Press, Cambridge (2009)

9. Mal'cev, A.I.: Iterative algebras and Post's varieties. Algebra Logika 5, 5-24 (1966) (in Russian)

10. Petrich, M.: Inverse Semigroups. Wiley, New York (1984)

11. Romanowska, A., Smith, J.D.H.: Modes. World Scientific, Singapore (2002) 